\title{
INSTABILIDADE DE ARMADURAS LONGITUDINAIS EM PILARES DE CONCRETO ARMADO
}

Salete Souza de Oliveira Buffoni ${ }^{1}$

Raul Rosas e Silva ${ }^{2}$

Resumo: A flambagem das armaduras longitudinais em pilares de concreto armado pode ocorrer na região entre dois estribos consecutivos, ou pode envolver um certo número de estribos. As normas de projeto existentes não fornecem uma metodologia apropriada para o dimensionamento dos estribos em diferentes situações. O presente trabalho tem por objetivo desenvolver uma formulação que permita analisar a flambagem das armaduras longitudinais em pilares de concreto armado submetidos a carregamento axial levando em conta o espaçamento entre os estribos, o diâmetro e arranjo dos estribos na seção transversal e o diâmetro das armaduras longitudinais. Para este propósito um método analítico para a avaliação da flambagem da armadura longitudinal é proposto, considerando-se as barras longitudinais restringidas pela rigidez axial ou à flexão dos estribos. Admite-se que a armadura longitudinal funciona como uma coluna esbelta. $\mathrm{O}$ presente trabalho trata a coluna com um ou mais modos de deformação, incluindo certas não-linearidades. São fornecidos cargas críticas e caminhos pós-críticos para tais casos. Como resultado deste estudo, apresenta-se uma proposta para dimensionamento racional dos estribos que permite estudar diferentes alternativas em um ábaco de utilização simples para projeto. Apresentam-se comparações com resultados experimentais da literatura em pilares de concreto armado. Isto permite uma avaliação crítica dos desenvolvimentos teóricos realizados e da forma proposta de dimensionamento racional dos estribos.

Palavras-chave: Flambagem; armaduras longitudinais e transversais; pilares; concreto armado.

\begin{abstract}
Buckling of longitudinal reinforcement in reinforced concrete columns may occur in the region between two consecutive ties, or may involve a number of ties. The existing design code specifications do not provide an appropriate methodology for the design of the transversal reinforcement in different situations. The main objective of the present work is to develop a formulation to allow to analyze the buckling of longitudinal bars in reinforced concrete columns taking into account the tie spacing, the diameter and arrangement of the ties in the cross section and the longitudinal bar diameter. For this purpose an analytical method for the evaluation of the buckling load of longitudinal bars is described, as a function of the constraint imposed by the axial or flexural stiffness of the stirrups. The longitudinal bar is considered as a column deforming according to thin beam theory. The theoretical analysis considers the column with one or more deformation modes, with some degree of nonlinearity, including the analysis of post-critical equilibrium paths. As a result of this study, rational criteria for spacing and sizing of transversal reinforcement are derived, allowing studying different alternatives in an abacus of simple use for design. Several comparisons with the results obtained experimentally by other authors in reinforced concrete columns are presented, allowing for an evaluation of the validity of the theoretical developments and the rational design methodology proposed herein.
\end{abstract}

Key words: Buckling; longitudinal and transverse reinforcement; reinforced concrete columns, columns; reinforced concrete.

\footnotetext{
${ }^{1}$ Universidade Federal Fluminense - UFF - E-mail: salete@vm.uff.br

${ }^{2}$ Pontifícia Universidade Católica do Rio de Janeiro - PUC-Rio - E-mail; raul@civ.puc-rio.br
} 


\section{INTRODUÇÃO}

O estudo da instabilidade das armaduras longitudinais em pilares de concreto armado tem atraído a atenção de vários pesquisadores nos últimos anos, entretanto a maioria dos estudos está restrita à análise da flambagem ocorrendo sempre entre dois estribos consecutivos como apresentam os trabalhos de Bresler \& Gilbert [3]. A flambagem da armadura longitudinal com a participação dos estribos no impedimento da mesma é um fenômeno pouco estudado na literatura.

O estudo experimental de pilares de concreto armado submetidos a carregamentos monotônicos ou cíclicos, com a finalidade de se visualizar o comportamento das armaduras longitudinais também tem atraído atenção de vários pesquisadores nos últimos quarenta anos dentre os quais cita-se os trabalhos de Pfister [10], Moehle \& Cavanagh [7] e Pantazopoulou [9] que apresentam em seus ensaios a flambagem das barras longitudinais em pilares de concreto armado envolvendo vários estribos.

Em face do exposto anteriormente, o presente trabalho tem como ponto de partida os resultados da pesquisa desenvolvida por Buffoni [4] que estuda a flambagem das armaduras longitudinais em pilares de concreto armado submetidos a carregamento axial levando em conta o espaçamento entre os estribos, o diâmetro e arranjo dos estribos na seção transversal e o diâmetro das armaduras longitudinais.

Para tal propósito um método analítico para a avaliação da flambagem da armadura longitudinal foi descrito, considerando-se as barras longitudinais restringidas pela rigidez axial ou à flexão dos estribos. Estudaram-se particularmente dois casos. No primeiro caso, considerouse a armadura longitudinal como uma coluna e os estribos como apoios discretos. No segundo caso, considerou-se a armadura longitudinal como uma coluna sobre base elástica, onde a base elástica é composta pelos estribos. Para se considerar ENGEVISTA, v. 8, n. 1, p. 49-64, junho 2006 o caso de emendas das barras na presente formulação, considerou-se a armadura livre em uma das extremidades. Considerou-se na modelagem um ou mais modos de deformação, incluindo-se a nãolinearidade geométrica. A partir destes resultados propõe-se um critério de projeto racional para a escolha do espaçamento, diâmetro dos estribos e arranjo das armaduras.

A dedução da formulação dos modelos matemáticos que serão abordados está fora do escopo deste artigo, procurando-se mostrar diretamente a aplicabilidade prática de tais modelos, por meio de comparações com resultados experimentais da literatura. Estimula-se o leitor a consultar Buffoni [4], que contempla de forma minuciosa as hipóteses e formulações inerentes a cada modelo.

\section{FORMULAÇÃO}

\subsection{Introdução}

O modelo matemático das armaduras longitudinais e transversais para a determinação da carga crítica apresenta-se na Figura 1, onde os estribos são representados esquematicamente como apoios elásticos intermediários unilaterais, cuja rigidez $K$ depende das características mecânicas e geométricas do aço.

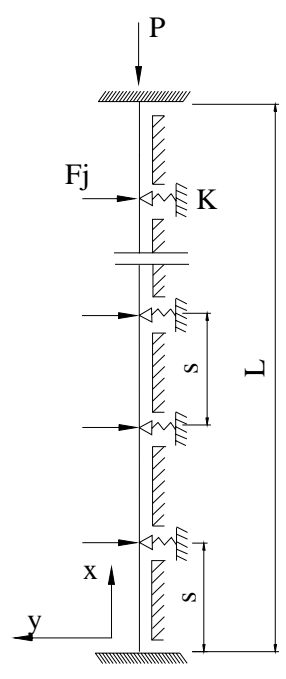

Figura 1- Modelo matemático da armadura longitudinal e transversal. 
O comprimento da coluna é designado por $L, s$ é o espaçamento entre estribos e $F_{j}$ são as forças correspondentes aos apoios elásticos $j$ e podem ser escritas como:

$$
F_{j}=K w_{j} \Rightarrow w_{j}=\frac{F_{j}}{K}
$$

onde $w_{j}$ é o deslocamento do apoio genérico. Neste trabalho, adotam-se as hipóteses usuais no campo da resistência dos materiais considerando-se que a coluna e o carregamento estão num plano de simetria e que a seção permanece plana e perpendicular ao eixo, antes e após as deformações.

\subsection{Energia Interna de Deformação e Energia Potencial da Viga-Coluna}

As matrizes de rigidez elástica e geométrica da coluna são obtidas a partir das energias de deformação e potencial, respectivamente. Toda a formulação matemática é realizada a partir da coluna de Euler, que é biapoiada e submetida a carregamento axial. Após a dedução das expressões que definem as matrizes de rigidez, adota-se um campo de deslocamentos que satisfaça as condições de contorno do modelo apresentado na Figura 1.

A energia interna de deformação fica representada pelo somatório da energia de membrana, $U_{m}$, proveniente das deformações da barra na direção axial mais a energia de flexão, $U_{f}$, proveniente das deformações devidas à flexão. Em geral, no problema da flambagem, as deformações devidas à flexão são bem mais importantes que as deformações axiais da barra e na formulação do problema não se leva em consideração a deformação axial, hipótese adotada na teoria inextensional de vigas como se verifica nos trabalhos de Bazant [2] e Dym \& Shames [5], a única parcela da energia interna considerada é a energia de flexão da viga, que é dada por:

$$
U \cong \int_{0}^{L} \frac{1}{2} E I w_{, x x}{ }^{2}\left[1+\frac{1}{2} w_{, x}{ }^{2}\right]^{2} d x
$$

Em virtude do modelo adotado, a energia de deformação dos estribos, os quais são considerados como molas é dada por:

$$
U_{i}=\frac{1}{2} K\left[\sum_{i=1}^{i=n} w(x=i s)\right]^{2}
$$

onde $w$ é o valor do deslocamento calculado nos pontos onde existem estribos e $n$ é o número de estribos envolvidos no modelo. Considerando-se que o apoio elástico discreto pode ser substituído por uma base elástica distribuída continuamente $k=K / s$, tem-se que o termo correspondente à energia de deformação dos estribos na expressão (3) pode ser avaliado como uma integral da seguinte forma:

$$
\frac{1}{2} K \sum_{i=1}^{i=n} w(x=i s)^{2} \cong \frac{1}{2} \int_{0}^{L} k w(x)^{2} d x
$$

O potencial das cargas externas é dado pelo produto da carga, $P$, e o encurtamento na extremidade da coluna $\Delta$, podendo ser expresso como:

$$
V_{p}=P \Delta \Rightarrow V_{p}=-P \int_{0}^{L}\left(\frac{1}{2} w_{, x}{ }^{2}+\frac{1}{8} w_{, x}^{4}\right) d x
$$

onde $P$ é o carregamento axial e o sinal negativo é devido o deslocamento se realizar no sentido contrário ao das forças. 


\subsection{Variáveis Adimensionais}

\section{- Caso discreto}

Quando se quer realizar uma análise paramétrica, é importante que a mesma seja realizada com eficiência, para isto foram feitas as seguintes mudanças de variáveis e escolhidos os seguintes parâmetros adimensionais:

$$
\begin{gathered}
\xi=\frac{x}{L} \quad 0 \leq \xi \leq 1 \quad w_{d}=\frac{w}{L} \\
\Gamma=\frac{P L^{2}}{E I} \quad \eta=\frac{K L^{3}}{E I}
\end{gathered}
$$

onde $\xi$ é o parâmetro adimensional do deslocamento axial, $w_{d}$ é o parâmetro adimensional do deslocamento lateral da coluna, $\Gamma$ é o parâmetro adimensional do carregamento axial e $\eta$ é o parâmetro adimensional da rigidez dos estribos.

\section{- Coluna sobre base elástica}

As variáveis adimensionais são as mesmas consideradas na expressão (6), com exceção do parâmetro adimensional da rigidez dos estribos que é dado por:

$$
\eta=\frac{k L^{4}}{E I}
$$

\subsection{Campo de Deslocamentos}

Observações experimentais mostram que a forma flambada da armadura longitudinal se parece muitas vezes com uma função senoidal sobre um período, o qual pode envolver vários espaçamentos entre estribos. Dessa forma, considerou-se a seguinte função para descrever o campo deslocamentos da coluna:

$$
\begin{aligned}
w(x)= & \sum_{m=1}^{\infty} A_{m}\left(-\frac{m \pi x}{L}+\frac{m \pi x^{2}}{L^{2}}\left(2+(-1)^{m}\right)-\right. \\
& \left.\frac{m \pi x^{3}}{L^{3}}\left(1+(-1)^{m}\right)+\sin \left(\frac{m \pi x}{L}\right)\right)
\end{aligned}
$$

Esse campo de deslocamentos combina funções que descrevem um comportamento simétrico com funções antissimétricas. A consideração de deformações apenas do tipo simétrico, pode se tornar um pouco distante do que acontece na realidade, pois se verifica em muitos casos que a flambagem pode envolver modos não simétricos. Na forma adimensional o campo de deslocamentos fica:

$$
\begin{aligned}
w_{d}(\xi)= & \sum_{m=1}^{j} a_{m}\left(-m \pi \xi+m \pi \xi^{2}\left(2+(-1)^{m}\right)-\right. \\
& \left.m \pi \xi^{3}\left(1+(-1)^{m}\right)+\sin (m \pi \xi)\right)
\end{aligned}
$$

\subsection{Integração ao Longo da Barra para Obtenção das Matrizes de Rigidez Elástica (Kf) e Rigidez Geométrica $(\mathrm{Kg})$}

A matriz de rigidez elástica representada por $K_{f}$ é obtida da energia de deformação expressa em (2), a matriz de rigidez que leva em consideração os apoios laterais, $K_{m}$, é obtida da energia de deformação (3) e (4) e a matriz de rigidez geométrica, $K_{g}$ é obtida a partir da energia da carga axial expressa em (5). Dessa forma, as expressões das matrizes de rigidez citadas considerando-se o campo de deslocamentos $w_{i}$ e $w_{j}$ para uma determinada condição de contorno são dadas a seguir.

\section{- Da energia de deformação}

$$
\begin{aligned}
K_{f_{i, j}}= & \int_{0}^{1}\left(w_{d, \xi \xi_{i}} w_{d, \xi \xi}+w_{d, \xi \xi_{i}} w_{d, \xi \xi_{j}} w_{d, \xi_{i}} w_{d, \xi_{j}}+\right. \\
& \left.\frac{1}{2} w_{d, \xi \xi_{i}} w_{d, \xi \xi} w_{d, \xi_{i}} w_{d, \xi_{j}} w_{d, \xi_{i}} w_{d, \xi_{j}}\right) d \xi
\end{aligned}
$$


- Da energia de deformação da mola

- Apoios distribuídos de forma discreta

$$
K_{m_{i, j}}=\eta \sum_{\xi=0}^{\xi=1} w_{d i} w_{d j}
$$

- Apoios distribuídos de forma contínua

$$
K_{m i, j}=\eta \int_{0}^{1} w_{d i} w_{d j} d \xi
$$

\section{- Da energia da carga axial}

$$
K_{g_{i, j}}=\int_{0}^{1}\left(w_{d, \xi_{i}} w_{d, \xi_{j}}+\frac{1}{4} w_{d, \xi_{i}} w_{d, \xi_{j}} w_{d, \xi_{i}} w_{d, \xi_{j}}\right) d \xi
$$

\subsection{Solução do Problema de Autovalores}

Considerando-se apenas a parcela quadrática nas expressões (10) a (13) temse o problema de autovalor, dado na seguinte expressão:

$$
K_{f}+K_{m}-\Pi K_{g}=0
$$

Resolvendo-se a equação (14) chega-se aos valores da carga crítica como a seguir:

$$
\Gamma=K_{g}^{-1}\left(K_{f}+K_{m}\right)
$$

\subsubsection{Parâmetros de Carga Crítica}

A partir da solução do problema de autovalores, foi possível encontrar os parâmetros de carga crítica para os casos discreto e contínuo. No caso discreto, considerou-se que o comprimento da armadura envolvida na flambagem varia de um a dezesseis espaçamentos entre estribos. Dessa forma, substitui-se o ENGEVISTA, v. 8, n. 1, p. 49-64, junho 2006 campo de deslocamentos com um ou mais graus de liberdade descrito em (9) nas expressões para a obtenção das matrizes de rigidez e resolve-se o problema de autovalores expresso em (15) obtendo-se assim os valores adimensionais para a carga de flambagem. Os valores para estes casos encontram-se no trabalho de Buffoni [4].

No caso contínuo, considerando-se a armadura longitudinal como uma coluna sobre base elástica, onde a base elástica é composta pelos estribos e substituindo-se o campo de deslocamentos descrito em (9) com um ou mais graus de liberdade apenas na parcela quadrática das expressões (10), (12) e (13) para obtenção das matrizes de rigidez e resolvendo-se o problema de autovalores expresso em (15) obtém-se os valores adimensionais para a carga crítica que se encontram em Buffoni [4]. A expressão (16) apresenta o parâmetro de carga crítica considerando-se apenas um termo na expansão modal.

$$
\Gamma=\frac{15 \pi^{6}-120 \pi^{4}+15 \pi^{2} \eta+\pi^{4} \eta-240 \eta}{5\left(5 \pi^{2}-48\right) \pi^{2}}
$$

\subsection{Consideração das Emendas das Barras da Armadura}

Para se considerar o caso de emendas das barras na presente formulação, considerou-se um modelo onde a armadura é engastada em uma das extremidades e livre na outra como o modelo apresentado na Figura 2. Dessa forma, todos os passos realizados para a coluna engastada nas extremidades foram feitos para o caso da presença de emendas com a finalidade de se conhecer o comportamento das armaduras quando uma das extremidades está livre. 


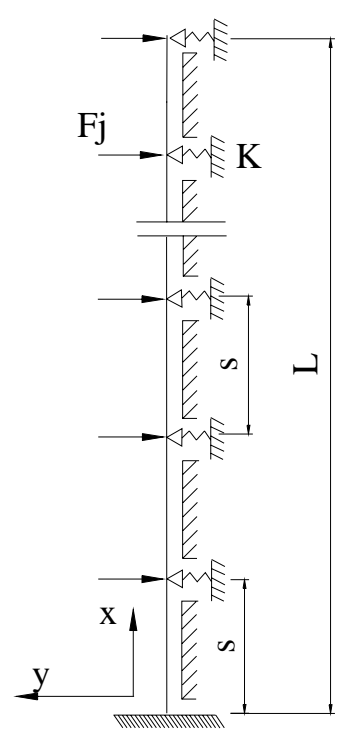

Figura 2- Modelo para consideração das emendas das barras da armadura.

\subsection{Cálculo do Parâmetro Adimensional de Rigidez, $\eta$}

O valor numérico do parâmetro adimensional de rigidez depende da rigidez dos estribos, $K$, do módulo de elasticidade da armadura longitudinal, $E$, do momento de inércia da armadura longitudinal, I e do espaçamento entre estribos, $s$ quando os estribos são considerados distribuídos continuamente ao longo da armadura.

\subsection{Cálculo da Rigidez dos Estribos, $\mathrm{K}$}

$O$ valor de $K$ é função das características mecânicas e da geometria do estribo. Considera-se nesta formulação os arranjos apresentados na Figura 3. Neste trabalho considera-se a carga axial concêntrica existindo condições de perfeita simetria, assim $K$ é calculada considerando-se os modelos simplificados da Figura 4.

O modelo da Figura 4.a está relacionado à barra localizada no centro de uma perna de estribos, as Figuras 4.b, 4.c, 4.d e 4.e estão relacionados às barras de canto, a Figura 4.f está relacionada à barra envolvida pelo estribo interior como mostra a Figura 3.c. A Figura 4 mostra também a força exercida pela barra longitudinal sobre o estribo na direção em que a flambagem pode ocorrer.

Para o modelo da Figura 4.a, a armadura longitudinal pode ser considerada como impondo uma carga concentrada no meio do vão de uma viga fixa nas extremidades e a expressão para a rigidez do estribo é dada por:

$$
K=\frac{192 E I_{t}}{b^{3}}
$$

Para o modelo da Figura 4.b a expressão para a rigidez do estribo fica:

$$
K=\frac{E A_{t}}{b}
$$

onde $E$ é o modulo de elasticidade da armadura longitudinal e $A_{t}$ é a área do estribo. Um resultado análogo é obtido para o modelo da Figura 4.c. Para os modelos das Figuras 4.d e 4.e, obtém-se um valor similar, porém o valor de $b$ seria $b / 2$. Quando o arranjo das armaduras na seção é semelhante a Figura 3.b, as barras localizadas no centro de uma perna de estribos são as primeiras a flambarem, se o arranjo for do tipo mostrado na Figura 3.c, as primeiras barras a flambarem são as barras do vértice, pois as barras do centro de uma perna envolvidas por um outro estribo possui uma rigidez muito maior do que a rigidez das barras de canto.

Nota-se que a armadura transversal pode oferecer diferentes contribuições para a resistência à flambagem das barras longitudinais. As barras longitudinais localizadas no canto dos estribos são restringidas por uma rigidez extensional e aquelas barras localizadas na perna de um estribo são restringidas apenas pela rigidez a flexão.

O módulo de elasticidade longitudinal a ser considerado é o módulo de elasticidade instantâneo, porém utiliza-se o módulo de elasticidade reduzido quando o mesmo é conhecido. 


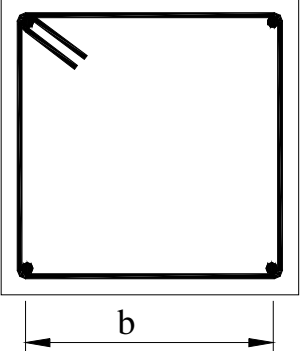

a)

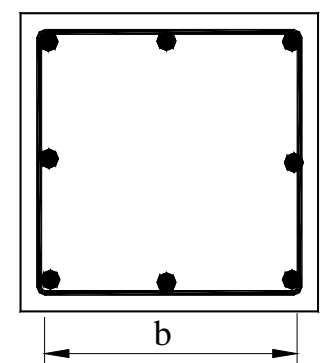

b)

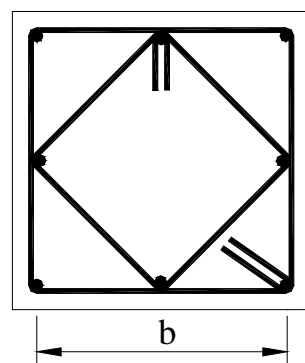

c)

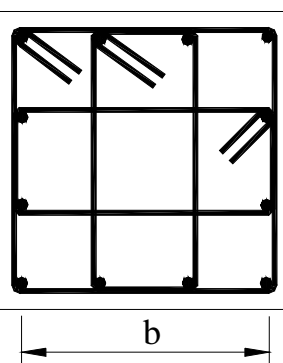

d)

Figura 3- Arranjos da armadura na seção transversal.

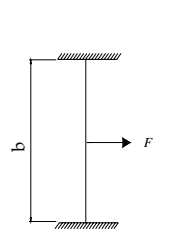

a)

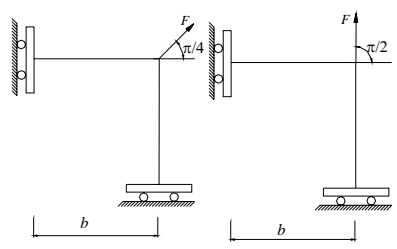

b)

c)

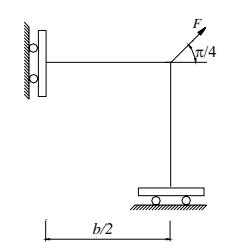

d)

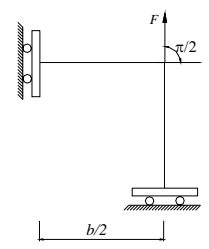

e)

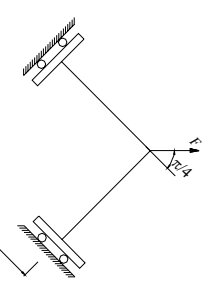

f)

Figura 4- Modelos simplificados para cálculo da rigidez $K$.

\section{CURVAS $\Gamma$ vs. $\eta$ PARA $O$ CÁLCULO DO ESPAÇAMENTO E DIÂMETRO DOS ESTRIBOS}

A Figura 5 apresenta a curva que relaciona o parâmetro de carga crítica da coluna, $\Gamma$ e o parâmetro de rigidez dos estribos, $\eta$ com as contribuições de vários modos para a carga de flambagem. Nota-se que este gráfico apresenta uma curva onde a armadura está fixa nas extremidades e outra curva onde uma das extremidades está livre para simular a presença de emendas das barras da armadura.

Verifica-se que a partir de um alto nível de rigidez, a carga de flambagem cresce quase que linearmente com o aumento da rigidez dos estribos. A partir deste gráfico será apresentado um método para o cálculo do espaçamento e rigidez dos estribos em um pilar de concreto armado.

\subsection{Curvas $\Gamma$ vs. $\eta$ Modificadas}

A curva da Figura 5 é válida para qualquer tipo de seção considerada. Conforme o objetivo do projeto, basta introduzir os respectivos valores de $\Gamma$ ou $\eta$ para cada caso em particular. Por exemplo, para a seção da Figura 3.b, substituindo-se o valor da rigidez dos estribos, $K$, da expressão (17) no valor do parâmetro adimensional de rigidez, $\eta$,expresso em (7) obtém-se o seguinte valor:

$$
\eta=\frac{192 \phi_{t}^{4} L^{4}}{b^{3} s \phi_{l}^{4}}
$$

Por outro lado, substituindo-se o momento de inércia da armadura longitudinal na expressão de $\Gamma$ tem-se:

$$
\Gamma=\frac{64 P L^{2}}{E \pi \phi_{l}^{4}}
$$

Dessa forma, é possível alterar os valores de $\Gamma$ e $\eta$ das expressões (19) e (20) e criar novos parâmetros com a finalidade de facilitar os cálculos dos exemplos que serão apresentados. As variáveis adimensionais $\Gamma$ e $\eta$ foram modificadas da seguinte forma: 


$$
\begin{aligned}
& \eta_{1}=\frac{\eta}{192}=\frac{\phi_{t}^{4} L^{4}}{b^{3} s \phi_{l}^{4}} \\
& \Gamma_{1}=\frac{\Gamma \pi}{64}=\frac{P L^{2}}{E \phi_{l}^{4}}
\end{aligned}
$$

Portanto a ordenada e abscissa dos gráficos da Figura 5 são alteradas multiplicando-se as mesmas pelos fatores, $\frac{\pi}{64}$ e $\frac{1}{192}$, respectivamente. Dessa forma, obtém-se o gráfico da Figura 6 com os parâmetros de carga e rigidez menores.

Dependendo do arranjo dos estribos na seção transversal, obtém-se um valor diferente para a rigidez, $K$ e para o parâmetro adimensional da rigidez dos apoios laterais, $\eta$.

\subsection{Considerações Sobre a Carga de Flambagem para Dimensionamento}

A carga de flambagem deve ser sempre maior que a carga de escoamento em compressão por um fator $\gamma>1$, isto para garantir 0 uso de $P_{y}=f_{y} A_{s}$ no dimensionamento inicial, como é feito usualmente. No dimensionamento usual, busca-se otimizar o projeto tomando $\gamma=1$, entretanto, é pouco recomendável do ponto de vista de segurança em regime póscrítico. Adotou-se no presente trabalho os valores de $\gamma=1,2$.

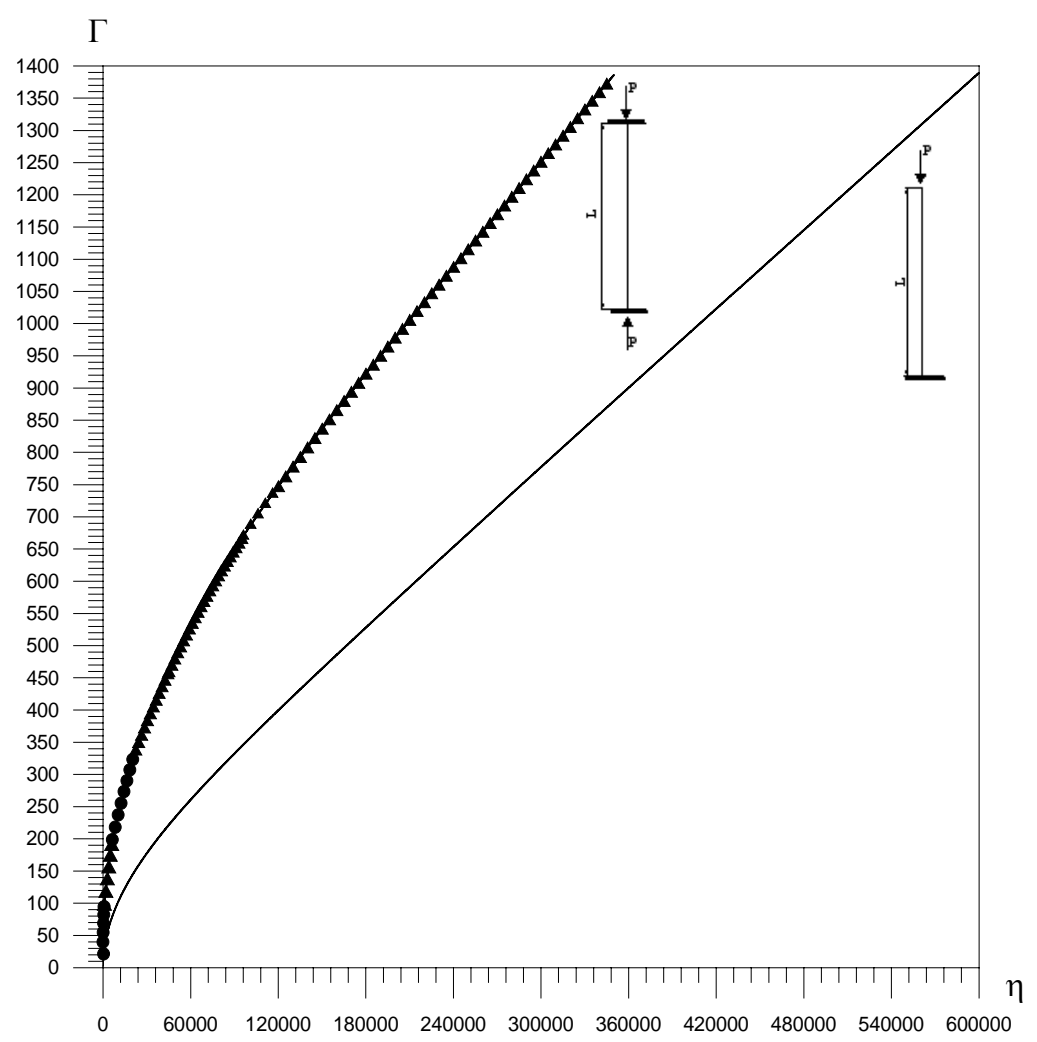

Figura 5- Parâmetro de carga vs. Parâmetro de rigidez da coluna com seis graus de liberdade. 


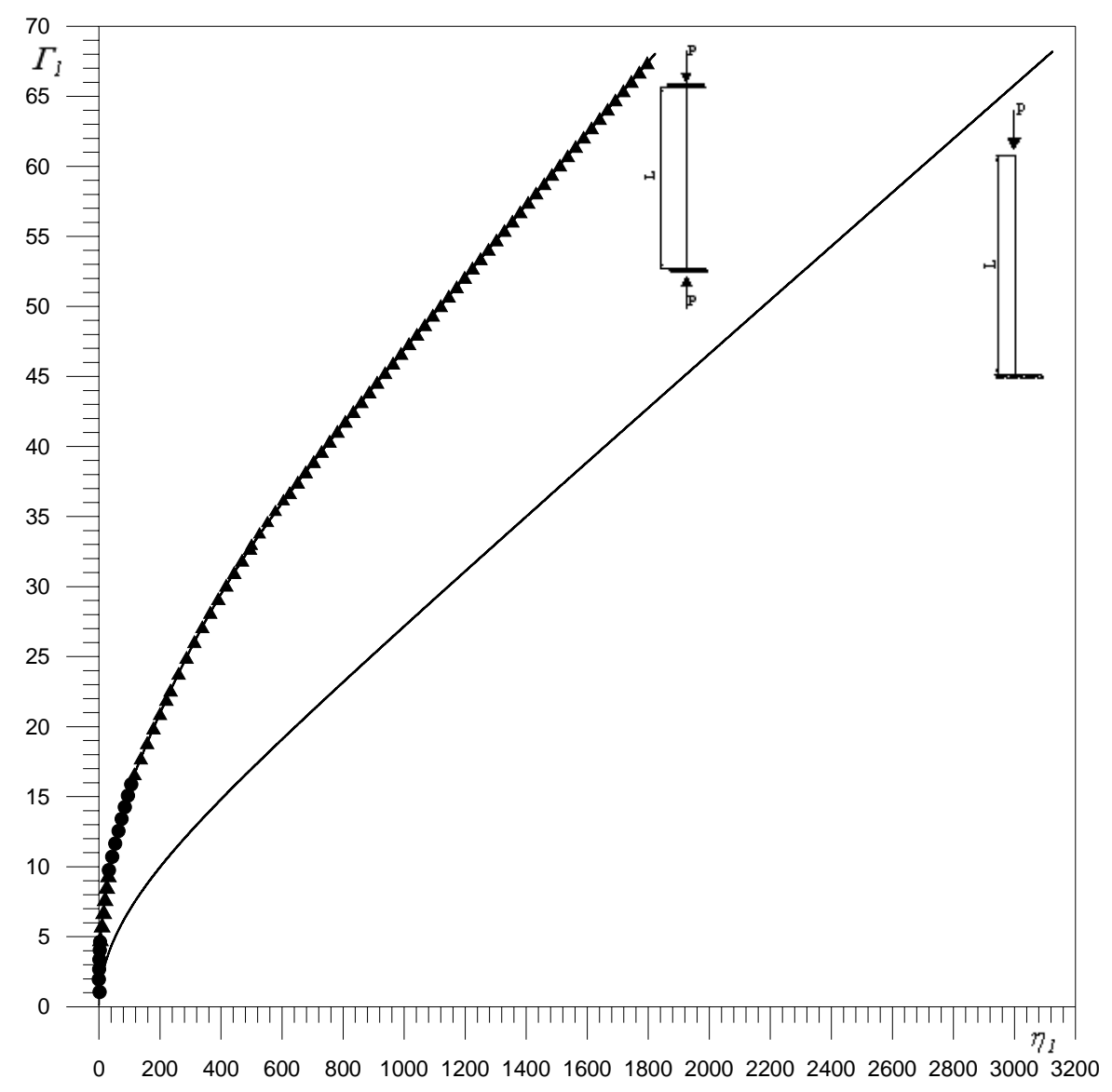

Figura 6 - Parâmetro de carga vs. Parâmetro de rigidez da coluna com seis graus de liberdade.

\section{SEQÜÊNCIA DE PROJETO COM A UTILIZAÇÃO DAS CURVAS $\Gamma$ vs. $\eta$}

Uma seqüência possível de projeto com o uso dos gráficos $\Gamma$ vs. $\eta$, seria como se mostra a seguir:

1- O valor de $b$ vem da geometria da peça;

2- O diâmetro da armadura longitudinal, $\phi_{l}$ é usualmente determinado pelo projetista;

3- Busca-se uma carga de flambagem, $P_{c r}=\gamma \cdot P_{y}$ e assim obtém-se $\Gamma_{1}$;

4- Com o valor de $\Gamma_{1}$ entra-se na ordenada do gráfico $\Gamma_{1}$ vs. $\eta_{1}$ e descobre-se o $\eta_{1}$ necessário na abscissa. Como já se dispõe dos valores de $b$ e $\phi_{l}$, as variáveis de projeto serão o espaçamento entre os estribos, $s$ e o diâmetro dos estribos, $\phi_{t}$, os quais serão calculados e adotados de forma compatível com os limites das normas de projeto existentes. Caso o espaçamento resulte muito pequeno, ou o diâmetro muito grande, é necessário reduzir $b$ ou usar estribos suplementares.

\subsection{Cálculo do Diâmetro e Espaçamento entre Estribos para os Pilares Descritos no Trabalho de Queiroga \& Giongo [12]}

Pretende-se analisar os pilares de seção quadrada cujo arranjo das armaduras na seção transversal apresenta-se na Figura 7 e o resumo das características dos pilares apresenta-se na Tabela 1. Os pilares P1, P4 e P6 foram selecionados para a realização dos testes numéricos. 


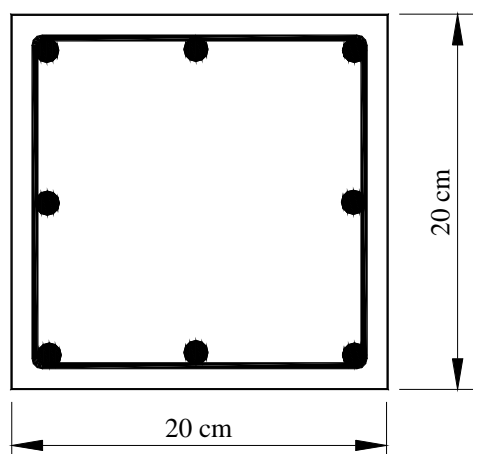

Figura 7- Seção e arranjo das armaduras nos pilares ensaiados por Queiroga [11].

Tabela 1 - Resumo das características dos pilares ensaiados por Queiroga [11].

\begin{tabular}{|l|c|c|c|c|c|c|}
\hline Pilar & $\begin{array}{c}\text { L } \\
(\mathrm{cm})\end{array}$ & $\begin{array}{c}\text { Arm. } \\
\text { Long } \\
\cdot\end{array}$ & $\begin{array}{c}A_{s} \\
\left(\mathrm{~cm}^{2}\right)\end{array}$ & $\begin{array}{c}\text { Arm. } \\
\text { Trans. }\end{array}$ & $\begin{array}{c}c \\
\mathrm{~mm}\end{array}$ & $\begin{array}{c}f_{c} \\
(\mathrm{MPa})\end{array}$ \\
\hline P1 & 120 & $8 \phi 12,5$ & 1,25 & $\phi 6,3 c / 15$ & 17,5 & 59,60 \\
\hline P4 & 120 & $8 \phi 12,5$ & 1,25 & $\phi 6,3 c / 10$ & 17,5 & 53,40 \\
\hline P6 & 120 & $8 \phi 12,5$ & 1,25 & $\phi 6,3 c / 5$ & 17,5 & 55,90 \\
\hline
\end{tabular}

Os valores calculados para o diâmetro e espaçamento entre os estribos utilizando o critério proposto no item 4 apresentam-se na Tabela 2. O procedimento completo de cálculo apresenta-se em Buffoni [4]. A seguir apresentam-se os passos realizados para o pilar P1.

\section{- Pilar P1}

As propriedades da armadura são:

$$
\begin{array}{ll}
f_{y}=502 \mathrm{~N} / \mathrm{mm}^{2} & \phi_{l}=12,5 \mathrm{~mm} \\
L=1200 \mathrm{~mm} & b=139,9 \mathrm{~mm} \\
E=210000 \mathrm{~N} / \mathrm{mm}^{2} & A_{s}=125 \mathrm{~mm}^{2} \\
P_{y}=f_{y} A_{s}=5,46 \times 10^{4} \mathrm{~N} & \mathrm{~s}=150 \mathrm{~mm}
\end{array}
$$

Pretende-se calcular o diâmetro e espaçamento entre estribos para $\gamma=1,2$ considerando-se a armadura sem emendas.
Assim, busca-se uma carga de flambagem, $P_{c r}=\gamma \cdot P_{y}$ e obtém-se $\Gamma_{1}$

$$
\begin{aligned}
& P_{c r}=\gamma \cdot P_{y}=65,48 \mathrm{kN} \Rightarrow \Gamma_{1}=\frac{P_{c r} \cdot L^{2}}{E \cdot \phi_{l}^{4}} \\
& \Rightarrow \Gamma_{1}=18,39 \Rightarrow \eta_{1}=149,10
\end{aligned}
$$

O valor de $\eta_{1}$ encontrado na expressão (22) foi obtido entrando-se com o valor de $\Gamma_{1}$ na ordenada do gráfico apresentado na Figura 6 e o valor necessário de $\eta_{1}$ encontra-se na abscissa correspondente a $\Gamma_{1}$. A partir da expressão (21) tem-se que:

$$
\frac{\phi_{t}^{4}}{s}=\frac{\eta_{1} \cdot b^{3} \cdot \phi_{l}^{4}}{L^{4}}=4,81
$$

A Tabela 2 apresenta os valores calculados para os pilares P4 e P6 que foram calculados da mesma forma que 0 pilar P1. Na linha correspondente ao pilar $\mathrm{P} 1$, os valores encontrados para o diâmetro, considerando-se um espaçamento $s=150 \mathrm{~mm}$ foi aproximadamente $\phi_{t}=5,2 \mathrm{~mm}$. Nota-se que estes valores são encontrados a partir do modo de flambagem que poderia inclusive envolver vários estribos num estado limite. A Tabela 2 apresenta os valores comerciais para o diâmetro do estribo.

Verifica-se também que quando se diminui o espaçamento entre estribos, 0 valor do diâmetro dos estribos poderia ser menor. A Tabela 2.b apresenta o caso onde uma das extremidades da armadura está livre, simulando o caso de emendas das barras da armadura.

Nesse caso, os valores encontrados para o diâmetro dos estribos são mais altos, visto que um certo valor do parâmetro de carga corresponde a valores mais altos de rigidez para o caso onde uma das extremidades da armadura está livre e, portanto, valores mais altos para o diâmetro dos estribos são necessários. 
De acordo com a NBR 6118/2003 o valor do espaçamento deve ser tal que em um estado limite a flambagem ocorreria entre dois estribos consecutivos. A Tabela 3 apresenta os valores limites para o pilar $\mathrm{P} 1$, do espaçamento e diâmetro dos estribos descritos em diversas normas de projeto de estruturas de concreto.

Verifica-se que o espaçamento máximo entre estribos fica em torno de $150 \mathrm{~mm}$ para um diâmetro dos estribos maior ou igual a $5 \mathrm{~mm}$. De acordo com as diversas normas, estes valores consideram no estado limite último, que a flambagem da armadura longitudinal ocorreria no máximo em uma meia-onda entre estribos, pois se considera que o comprimento de flambagem é o próprio espaçamento entre dois estribos.

Tabela 2- Cálculo do diâmetro e espaçamento entre estribos para os pilares de Queiroga [11] a partir da formulação proposta.

a) Armadura fixa nas extremidades

\begin{tabular}{|c|c|c|c|c|c|c|}
\hline \multicolumn{2}{|c|}{ Queiroga [11] } & \multicolumn{5}{|c|}{$\begin{array}{c}\text { Formulação Proposta } \\
\text { Armadura fixa nas extremidades } \\
\qquad \gamma=1,2\end{array}$} \\
\hline \multirow[b]{2}{*}{ Pilar } & \multirow{2}{*}{$\begin{array}{c}\text { Arm. } \\
\text { Transv. }\end{array}$} & \multirow[b]{2}{*}{$\Gamma_{1}$} & \multirow[b]{2}{*}{$\eta_{1}$} & \multicolumn{3}{|c|}{ Arm. Transv. } \\
\hline & & & & $\begin{array}{c}\mathrm{s} \\
\mathrm{mm}\end{array}$ & $\begin{array}{c}\phi_{t} \\
m m\end{array}$ & $\begin{array}{l}\phi_{\text {tcom }} \\
\mathrm{mm}\end{array}$ \\
\hline $\mathrm{P} 1$ & $\phi 6,3 c / 15$ & 18,39 & 149,1 & 150 & 5,20 & 6,3 \\
\hline P4 & $\phi 6,3 c / 10$ & 18,39 & 149,1 & 100 & 4,68 & 5 \\
\hline P6 & $\phi 6,3 c / 5$ & 18,39 & 149,1 & 50 & 3,94 & 5 \\
\hline
\end{tabular}

Obs: Para o diâmetro do estribo, $\phi_{t}=6,3 \mathrm{~mm}$ encontra-se um espaçamento máximo de $328 \mathrm{~mm}$.

b) Armadura Livre em Uma das Extremidades

\begin{tabular}{|c|c|c|c|c|c|}
\hline \multicolumn{2}{|c|}{ Queiroga [11] } & \multicolumn{3}{|c|}{$\begin{array}{c}\text { Formulação Proposta } \\
\text { Armadura livre em uma das extremidades } \\
\gamma=1,2\end{array}$} \\
\hline \multirow{2}{*}{ Pilar } & \multirow{2}{*}{$\Gamma_{1}$} & \multirow{2}{*}{$\eta_{1}$} & $s$ & \multicolumn{3}{|c|}{ Arm. Transv. } \\
\cline { 4 - 7 } & & & $m m$ & $\begin{array}{c}\phi_{t} \\
m m\end{array}$ & $\begin{array}{l}\phi_{\text {tcom }} \\
m m\end{array}$ \\
\hline P1 & 18,39 & 566,30 & 150 & 7,23 & 8 \\
\hline P2 & 18,39 & 566,30 & 100 & 6,54 & 6,3 \\
\hline P3 & 18,39 & 566,30 & 50 & 5,50 & 8 \\
\hline
\end{tabular}

Obs: Para o diâmetro do estribo, $\phi_{t}=6,3 \mathrm{~mm}$ encontra-se um espaçamento máximo de $86 \mathrm{~mm}$. 
Tabela 3 - Valores limites para o espaçamento e diâmetro dos estribos para o pilar P1 de Queiroga [11] obtidos de normas de projeto.

\begin{tabular}{|c|c|c|c|}
\hline \multicolumn{3}{|c|}{ Valores Máximos fixados em Normas } \\
flambagem com uma meia-onda entre dois \\
estribos \\
\hline \multicolumn{3}{|c|}{ NBR 6118} \\
ACI 318 & \multicolumn{2}{c|}{2003} \\
\hline$s \leq 16 \phi_{l}$ & $\phi_{t} \geq 10$ & $\begin{array}{c}s \leq 12 \phi_{l} \\
(m m)\end{array}$ & $\begin{array}{c}\phi_{t} \geq 5 \\
(\mathrm{~mm})\end{array}$ \\
\hline$(\mathrm{mm})$ & $(\mathrm{mm})$ & 150 & 5 \\
\hline 150 & 10 &
\end{tabular}

\subsection{Aplicações para Pilares com Seções de Grandes Dimensões}

Aplica-se o critério proposto a um pilar de concreto armado com seção transversal retangular $25 \mathrm{~cm} \times 110 \mathrm{~cm}$, altura livre de $350 \mathrm{~cm}$, com resistência à compressão do concreto $20 \mathrm{MPa}$ e do aço de $500 \mathrm{MPa}$. A armadura longitudinal consiste de 22 barras de $16 \mathrm{~mm}$ de diâmetro, e o cobrimento é de $3 \mathrm{~cm}$, conforme apresentado na Tabela 4. Nomeou-se este pilar de P1 e se apresenta a seguir alguns casos de variações nos arranjos das armaduras na seção transversal.
Tabela 4 - Resumo das características do pilar P1.

\begin{tabular}{|c|c|c|c|c|c|c|}
\hline Pilar & $\begin{array}{c}B \\
\mathrm{~cm}\end{array}$ & $\begin{array}{c}H \\
\mathrm{~cm}\end{array}$ & $\begin{array}{c}L \\
\mathrm{Cm}\end{array}$ & $\begin{array}{l}\text { Arm. } \\
\text { Long }\end{array}$ & $\begin{array}{c}\mathrm{C} \\
\mathrm{cm}\end{array}$ & $\begin{array}{c}f_{y k} \\
\mathrm{Mpa}\end{array}$ \\
\hline P1 & 25 & 110 & 350 & $22 \phi 16$ & 3 & 500 \\
\hline
\end{tabular}

\section{- Caso 1}

Considera-se o arranjo das armaduras na seção transversal apresentado na Figura 8. Para o cálculo da rigidez $K$ dos estribos, adotou-se o modelo da Figura 9, onde a perna do estribo é considerada como uma viga fixa nas extremidades. A flexibilidade do estribo associada a cada barra pode ser obtida aplicando-se uma carga transversal concentrada unitária isoladamente em cada ponto central da barra. Nota-se que isto corresponde a admitir-se que o início da flambagem se dá na barra menos restringida. Assim, avalia-se o ponto crítico como correspondente a uma barra que contribui com a menor rigidez do estribo. Para o modelo da Figura 9 isto ocorre no centro da viga, e neste caso a rigidez fica sendo:

$$
K=\frac{38.4 E I_{t}}{b^{3}}
$$

onde o vão livre de flexão é $b=1100-2(30+5)-16=1014 \mathrm{~mm}$

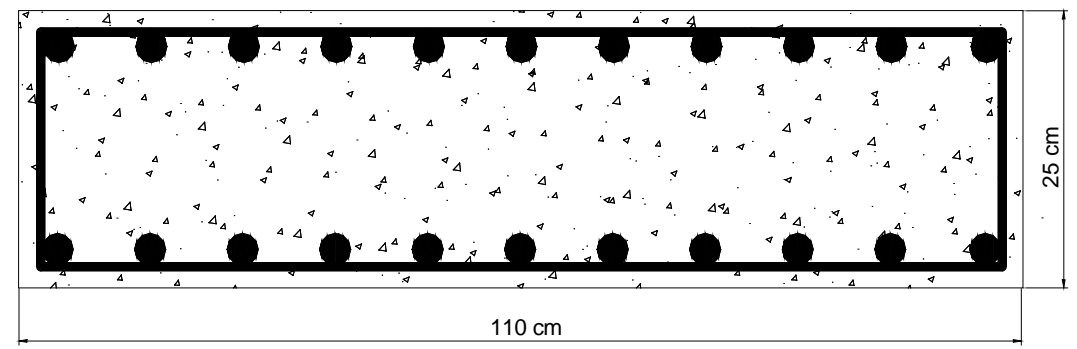

Figura 8- Caso 1: Arranjo das armaduras na seção transversal do pilar P1.

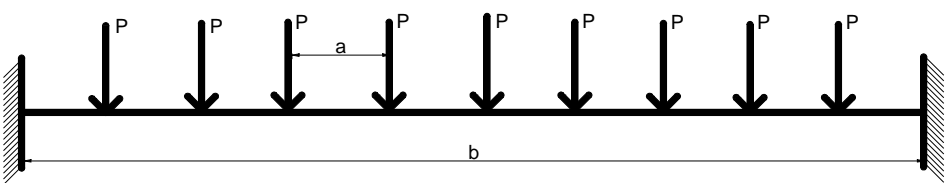

Figura 9- Modelo simplificado para cálculo da rigidez $K$ referente ao caso 1. 
Supôs-se inicialmente que o diâmetro do estribo é igual a $5 \mathrm{~mm}$. Considerando os estribos como base elástica e substituindose $k=K / s$ no parâmetro $\eta$ chega-se a:

$$
\begin{gathered}
\eta=\frac{k L^{4}}{E I}=\frac{38,4 E I_{t} L^{4}}{b^{3} s E I}=\frac{38,4 \phi_{t}^{4} L^{4}}{b^{3} s \phi_{l}^{4}} \\
\Rightarrow \frac{\phi_{t}^{4}}{s}=\frac{\eta b^{3} \phi_{l}^{4}}{38,4 L^{4}}=\frac{192 \eta_{1} b^{3} \phi_{l}^{4}}{38,4 L^{4}}
\end{gathered}
$$

Pretende-se calcular o diâmetro e espaçamento entre estribos para $\gamma=1,2$ considerando-se a armadura sem emendas. Dessa forma, busca-se uma carga de flambagem, $P_{c r}=\gamma \cdot P_{y}$ e obtém-se $\Gamma_{1}$ e $\eta_{1}$.

$$
\begin{aligned}
& P_{c r}=\gamma \cdot P_{y}=104,4 \mathrm{kN} \Rightarrow \Gamma_{1}=\frac{P_{c r} \cdot L^{2}}{E \cdot \phi_{1}^{4}} \\
& \Rightarrow \Gamma_{1}=92,93 \Rightarrow \eta_{1}=2802,08
\end{aligned}
$$

A partir da expressão (26) tem-se:

$$
\frac{\phi_{t}^{4}}{s}=6,38 \times 10^{3}
$$

Estipularam-se alguns valores para o espaçamento entre estribos de acordo com os limites impostos pela NBR 6118/2003. Para que a base elástica possa impedir a flambagem da armadura longitudinal os diâmetros encontrados apresentam-se na Tabela 5. Os valores encontrados para o diâmetro do estribo foram altos, visto que este modelo é bastante flexível.
Tabela 5 - Dimensionamento dos estribos para o caso 1.

\begin{tabular}{|c|c|c|c|}
\hline$s(\mathrm{~mm})$ & 190 & 150 & 50 \\
\hline$\phi_{t}(\mathrm{~mm})$ & 33,18 & 31,28 & 23,77 \\
\hline
\end{tabular}

\section{- Caso 2}

O modelo da Figura 10 se apresentou o mais favorável e econômico em termos de dimensionamento dos estribos. O modelo simplificado para o cálculo de $K$ apresentase na Figura 11, onde $\mathrm{o}$ valor de $b=338 \mathrm{~mm}$, sendo $K$ dada por:

$$
K=\frac{6 b E I_{t}}{a^{3}(2 b-3 a)}
$$

A expressão para o cálculo do dimensionamento dos estribos obtida a partir das expressões de $\eta$ e $K$ apresentadas em (7) e (28), respectivamente é dada por:

$$
\frac{\phi_{t}^{4}}{s}=\frac{32 \eta_{1} \phi_{l}^{4} a^{3}(2 b-3 a)}{b L^{4}}
$$

Os resultados apresentam-se na Tabela 6 para os valores de $\gamma=1,0$ e $\gamma=1,2$. verificou-se através dos cálculos que ao se definir o diâmetro do estribo como $\phi_{t}=6,3 \mathrm{~mm}$, o espaçamento entre estribos fica bem maior do que o limite superior registrado na NBR 6118/2003. 


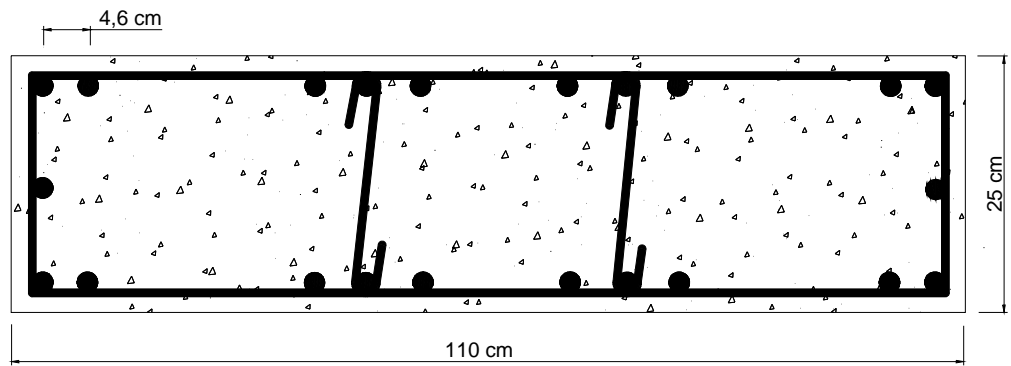

Figura 10- Caso 2: Arranjo das armaduras na seção transversal do pilar P1.

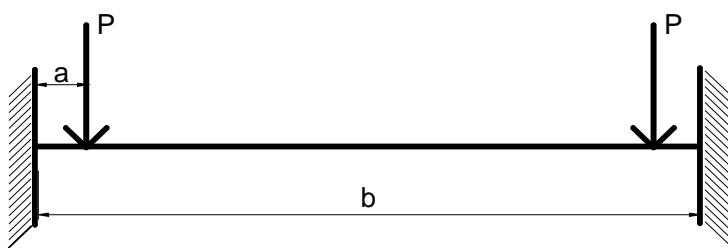

Figura 11- Modelo simplificado para cálculo da rigidez $K$ referente ao caso 2.

Tabela 6 - Dimensionamento dos estribos para o caso 2.

a) Cálculo do Diâmetro dos estribos.

\begin{tabular}{|c|c|c|}
\hline & \multicolumn{2}{|c|}{$\phi_{t}(\mathrm{~mm})$} \\
\hline & $\gamma(\mathrm{mm})$ & \multicolumn{2}{|c|}{} & $\gamma=1,0$ \\
\hline 190 & 5,48 & 5,83 \\
\hline 150 & 5,16 & 5,49 \\
\hline 50 & 3,92 & 4,17 \\
\hline
\end{tabular}

b) Cálculo do espaçamento.

\begin{tabular}{|c|c|c|}
\hline & \multicolumn{2}{|c|}{$s(\mathrm{~mm})$} \\
$\phi_{t}(\mathrm{~mm})$ & \multicolumn{2}{|c|}{} \\
\hline & $\gamma=1,0$ & $\gamma=1,2$ \\
\hline 5 & 131 & 103 \\
\hline 6,3 & 331 & 259 \\
\hline
\end{tabular}

A partir deste modelo realizou-se um estudo para verificar a possibilidade de executá-lo com espaçamento duplo. Calculou-se a rigidez média efetiva da base elástica como sendo a média dos valores da rigidez $K$ calculada com estribos suplementares utilizando-se 0 modelo simplificado da Figura 11, que se apresenta na expressão (28), e a rigidez do modelo da Figura 10 sem estribos suplementares, como apresenta o modelo simplificado da Figura 12. O valor da rigidez, $K$, para o modelo da Figura 12, correspondente a uma das cargas mais próximas do centro que é dada por:

$$
K=\frac{54 b E I_{t}}{\left(b^{4}+3 b^{3} a-9 a^{2} b^{2}+18 a^{3} b-81 a^{4}\right)}
$$

O valor de $b=1014 \mathrm{~mm}$ e os resultados considerando-se a rigidez média da base elástica para a consideração de espaçamento duplo, com os valores de $\gamma=1,0$ e $\gamma=1,2$ apresentam-se na Tabela 7.

Tabela 7 - Caso2: Dimensionamento dos estribos considerando-se espaçamento duplo.

a) Cálculo do diâmetro dos Estribos

\begin{tabular}{|c|c|c|}
\hline \multirow{2}{*}{$s(\mathrm{~mm})$} & \multicolumn{2}{|c|}{$\phi_{t}(\mathrm{~mm})$} \\
\hline & $\gamma=1,0$ & $\gamma=1,2$ \\
\hline 190 & 6,51 & 6,93 \\
\hline 150 & 6,14 & 6,53 \\
\hline
\end{tabular}




\begin{tabular}{|l|l|l|}
\hline 50 & 4,96 & 4,96 \\
\hline
\end{tabular}

b) Cálculo do Espaçamento

\begin{tabular}{|c|c|c|}
\hline & $\gamma=1,0$ & $\gamma=1,2$ \\
\hline 5 & 66 & 51 \\
\hline 6,3 & 166 & 129 \\
\hline
\end{tabular}

\begin{tabular}{|l|c|}
\hline$\phi_{t}(\mathrm{~mm})$ & $s(\mathrm{~mm})$ \\
\hline
\end{tabular}

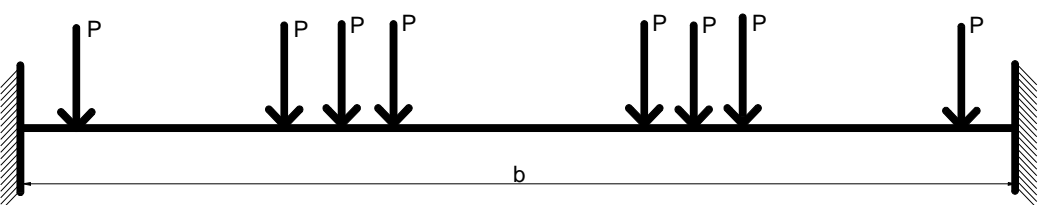

Figura 12- Modelo simplificado para cálculo da rigidez $K$ referente ao caso 2 sem estribos

\section{CONCLUSÕES}

A partir da obtenção dos gráficos que relacionam o parâmetro de carga da armadura longitudinal com o parâmetro de rigidez dos estribos, mostrou-se interessante propor uma seqüência de projeto, caracterizando $\quad 0$ dimensionamento racional dos estribos em pilares de concreto armado. Os exemplos mostram que o resultado de tal procedimento pode se tornar compatível com os resultados existentes em normas de projeto atuais, por uma calibragem adequada dos parâmetros envolvidos. Os valores calculados para o espaçamento e diâmetro dos estribos foram obtidos a partir da consideração de um modo de flambagem geral que poderia inclusive envolver vários estribos.

A partir dos resultados apresentados verifica-se que a armadura transversal pode oferecer diferentes contribuições para a resistência a flambagem das barras longitudinais. Foi mostrado, através de exemplos, que o procedimento adotado neste trabalho permite que se atinja a rigidez necessária, com diversas variáveis: espaçamento entre os estribos, diâmetro dos estribos, diâmetro da armadura longitudinal, uso de estribos suplementares, e reposicionamento das barras da armadura longitudinal. Estas duas últimas variáveis correspondem a ENGEVISTA, v. 8, n. 1, p. 49-64, junho 2006 variações no vão de flexão dos estribos.

Pode ainda ser considerada uma rigidez efetiva para o estribo como a média entre a rigidez calculada quando se consideram estribos suplementares e a rigidez calculada sem a consideração dos mesmos. Assim, dimensiona-se o estribo utilizando um espaçamento duplo, ou seja, a cada dois espaçamentos colocamse estribos suplementares, o que pode ser benéfico na hora da concretagem.

Em resumo, propõe-se um projeto racional de armadura transversal, com uso de considerações relacionadas a flambagem da armadura longitudinal. Um eventual maior consumo de armadura pode ser compensado por melhores condições de execução de pilares, devido à redução de armaduras suplementares.

\section{REFERÊNCIAS}

1. American Concrete Institute Committee 318. "Building Code Requirements for Structural Concrete (ACI 318-02) and Commentary (318R02)”, American Concrete Institute, Farmington Hills, Mich, 2002.

2. Bazant, Z. P.; Cedolin, L. Stability of Structures - Elastic, Inelastic, Fracture, and Damage theories. Oxford University Press, New York, 1991. 
3. Bresler, B.; Gilbert, P. H. Tie Requirements for Reinforced Concrete Columns. ACI Journal. Vol. 58, No. 5, p. 555-570, 1961.

4. Buffoni, S. S. O. Estudo da Flambagem de Armaduras Longitudinais em Pilares de Concreto Armado. Tese de Doutorado, Departamento de Engenharia Civil, PUC/Rio, 2004.

5. Dym, C. L.; Shames, I. H. Solid Mechanics - A Variational Approach. McGraw-Hill-Kogakusha, Ltd . Tokyo, 1973

6. James, F. P. Influence of Ties on the Behavior of Reinforced Concrete Columns. Journal of the American Concrete Institute. Proceedings Vol. 61, No. 5, p. 521-537, 1964.

7. Moehle, J. P.; Cavanagh, T. Confinement Effectiveness of Crossties in $R C$. Journal of Structural Engineering, Vol. 111, № 10, p. 2105-2120, 1985.

8. NBR-6118., 2003. Projeto e execução de obras de concreto armado.

9. Pantazopoulou, S. J. Detailing for Reinforcement Stability in RC Members. Journal of Structural Engineering, Vol. 124, № 6, ASCE, p. 623-632, 1998.

10. Pfister, J. F. Influence of Ties on the Behavior of Reinforced Concrete Columns. Journal of the American Concrete Institute, Vol. 61, No. 5, p. 521536, 1964.

11. Queiroga, M. V. M. Análise Experimental de Pilares de Concreto de Alto Desempenho Submetidos à Compressão Simples. Dissertação de Mestrado apresentada à Escola de Engenharia de São Carlos, EESC - USP, 1999.

12. Queiroga, M. V. M.; Giongo, J. S. Resistência e Ductilidade de Modelos de Pilares de Concreto de Alta Resistência Submetidas à Compressão Simples. IV Simpósio EPUSP sobre Estruturas de Concreto, SP, 2000. 